\title{
Conjunto de Redes Neurais Artificiais para Detecção Online de Fótons de Altas Energias
}

\author{
J. L. Marin ${ }^{1}$, E. F. de Simas Filho ${ }^{1}$, E. E. P. de Souza ${ }^{1}$, \\ B. S.M. Peralva ${ }^{2}$, J. V. F. Pinto ${ }^{3}$, M. V. de Araújo ${ }^{3}$, J. M. de Seixas ${ }^{3}$ \\ ${ }^{1}$ Laboratório de Sistemas Digitais, PPGEE/UFBA, ${ }^{2}$ PPGMVC/UERJ, \\ ${ }^{3}$ Laboratório de Processamento de Sinais, COPPE/POLI, UFRJ \\ ${ }^{1}$ Salvador, ${ }^{2}$ Nova Friburgo, ${ }^{3}$ Rio de Janeiro, Brasil \\ \{juan.lieber, eduardo.simas, edmar.egidio\}@ufba.br \\ bernardo.peralva@uerj.br \\ \{jodafons, micael.verissimo, seixas\}@lps.ufrj.br
}

\begin{abstract}
Resumo-A correta identificação de partículas é um dos principais objetivos de experimentos de física de altas energias. Devido a alta taxa de eventos no Grande Colisor de Hádrons (LHC), o experimento ATLAS tem empregado técnicas baseadas em aprendizado de máquina a fim de encontrar eventos raros em grandes massas de dados. Entre eles está o NeuralRinger, um conjunto classificador de rede neural projetado para detecção rápida de elétrons com base em anéis concêntricos de calorímetro (sistema de medição de energia). Nesse contexto, o presente trabalho propõe a adaptação desta técnica, que opera identificando elétrons, para a detecção rápida de fótons. Os resultados com dados simulados mostram a eficiência do método proposto no ambiente do experimento ATLAS.

Index Terms-Redes Neurais, NeuralRinger, Física de Altas Energias, Identificação de partículas
\end{abstract}

\section{IntRoduÇão}

Um dos maiores motores da humanidade é a eterna busca pelo conhecimento. A composição do universo, bem como sua origem, é até hoje objeto de grande interesse da comunidade científica. $\mathrm{O}$ estudo de partículas atômicas e subatômicas tem sido estudada através de colisores de partículas de alta energia [1]. O LHC (do inglês, Large Hadron Collider), construído no CERN (Centro Europeu para Pesquisa Nuclear), é o maior colisor de partículas em atividade, consistindo em um percurso aproximadamente circular de 27 quilômetros. Construído a 100 metros abaixo da superfície na fronteira franco-suíça, o LHC opera acelerando feixes de prótons, com velocidade próxima a da luz, colocando-os em colisão a cada $25 \mathrm{~ns}$, alcançando uma energia de $13 \mathrm{TeV}$. Com isso, é possível aumentar a probabilidade de ocorrência de partículas raras, que auxiliam em um melhor entendimento na composição do universo [2]. Por conta disso, um alto volume de dados é gerado por evento, cerca de $52 \mathrm{~TB} / \mathrm{s}$, sendo necessário um sistema de filtragem de eventos, reduzindo os dados apenas com os eventos de interesee. No experimento ATLAS (do inglês, A Toroidal LHC AparatuS) [3], o sistema de filtragem é divido em dois níveis em cascata, a fim de reduzir a latência entre as etapas de processamento. Esse sistema é também responsável por filtrar os eventos de interesse, dentro da grande massa de dados que é gerada durante as colisões, que são definidos por canais físicos extremamente raros.
Com essa alta taxa de produção de colisões ocorre o problema do empilhamento de sinais [4], que degrada o desempenho dos diversos métodos de identificação de partículas. Em especial, os fótons são de grande interesse do experimento, sendo a primeira observação do bóson de Higgs feita através da identificação de um par de fótons [5]. Recentemente, um novo decaimento deste mesmo bóson foi observado, sendo uma das partículas resultantes um fóton [6]. Usualmente, o ruído de fundo associado aos fótons é composto por jatos hadrônicos que podem falsear a assinatura de fótons.

$\mathrm{O}$ uso de técnicas de aprendizado de máquina vem se tornando uma constante na solução de diversos problemas de engenharia e instrumentação científica, principalmente em cenários onde há uma grande quantidade de dados e desejase encontrar um evento raro que possua características singulares. As redes neurais artificiais se mostram uma poderosa ferramenta na solução desse tipo de problema, fazendo uso de algoritmos de otimização e diferentes arquitetura de modelos [7]. O trabalho desenvolvido em [8] mostra a evolução do uso de redes neurais artificiais em experimentos de física de altas energias. Em [9] uma rede neural adversarial é usada para reduzir as incertezas sistemáticas na identificação de jatos hadrônicos. Já em [10], o uso de aprendizado profundo em redes neurais é utilizado para a busca de partículas exóticas em física de altas energias. O algoritmo NeuralRinger [11], que usa um modelo neural MLP (do inglês, Multi Layer Perceptron), foi desenvolvido para a discriminação elétron/jato no experimento ATLAS, no complexo de aceleração LHC. Este algoritmo, descrito detalhadamente na seção II-B, utiliza a informação de energia dos canais de leitura do calorímetro para montar anéis concêntricos e alimentar uma rede neural para performar a discriminação. Desde 2017, é a técnica padrão de detecção online de elétrons no ATLAS, para energias superiores a $15 \mathrm{GeV} 1$ [12].

Nesse contexto, o objetivo desse trabalho é adaptar a técnica NeuralRinger para a seleção rápida de fótons, fazendo uso do

\footnotetext{
${ }^{1} \mathrm{~A}$ unidade padrão de energia em física de altas energias é o eletron-volt $(\mathrm{eV})$. Essa unidade é definida como a quantidade de energia cinética para ganha por um elétron quando submetido a uma diferença de potencial de $1 \mathrm{~V}$ no vácuo
} 
mesmo procedimento adotado para o cenário de elétrons. O texto está organizado da seguinte forma: a Seção II descreve o experimento ATLAS, o seu sistema de seleção de eventos e o algoritmo NeuralRinger. Já a Seção III descreve a metodologia utilizada. A Seção IV discute os resultados e impactos do uso do NeuralRinger nas cadeias de fótons. As discussões são sumarizadas na Seção V

\section{O EXPERIMENTO ATLAS}

Figurando como maior experimento do LHC, o ATLAS [3]) foi um dos responsáveis, em 2012, pela descoberta do bóson de Higgs [5], enaltecendo seu papel nas principais descobertas científicas atuais. É composto por diversos subsistemas, ilustrados na Fig. 1, apoiando-se fortemente em medidas do calorímetro (subsistema de medição da energia das partículas) [13]. Nessa figura, pode-se observar o calorímetro eletromagnético, em amarelo, que é responsável por medir principalmente energia de partículas eletromagnéticas como elétrons e fótons. É divido em três camadas e possui aproximadamente 200 mil canais de leitura. Já o calorímetro hadrônico, em cinza, é responsável por prover medidas que auxiliam na caracterização de partículas hadrônicas, como prótons e píons, possuindo também três camadas e aproximadamente $10 \mathrm{mil}$ canais de leitura. O ATLAS adota um sistema de coordenadas cilíndrica, sendo as variáveis $\eta$ e $\phi$ as principais. ${ }^{2}$

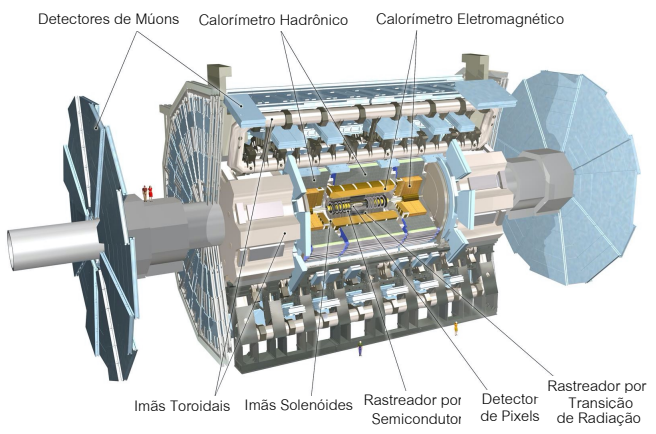

Figura 1: Experimento ATLAS e seus diversos subsistemas (adaptado de [3])

O ATLAS, através do seu sistema de seleção de eventos online (trigger), utiliza algoritmos para garantir que as partículas associadas a seu programa de pesquisa sejam corretamente identificadas [14].

\section{A. Seleção de Eventos no experimento ATLAS}

O método atual de seleção de fótons do experimento ATLAS opera aplicando cortes lineares em variáveis discriminantes, calculadas em diversas seções dos calorímetros eletromagnético e hadrônico [12]. Os eventos de interesse

\footnotetext{
${ }^{2} \mathrm{O}$ ATLAS usa um sistema de coordenadas baseado na mão direita, com sua origem no ponto de interação (PI) no centro do detector e o eixo $\mathrm{z}$ ao longo do feixe de prótons. O eixo x é direcionado a partir do PI até o centro do anel do LHC e o eixo y é perpendicular a este plano. As coordenadas cilíndricas $(r, \phi)$ são usadas no plano transverso, com $\phi$ sendo o ângulo azimutal em torno do eixo z. A pseudo rapidez é definida em termos do ângulo polar $\theta$ como $\eta \equiv-\ln [\tan (\theta / 2)]$.
}

do experimento ATLAS (trigger) passam por um sistema de seleção que é dividido em dois níveis [15]. O primeiro, L1, é implementado em hardware dedicado e é responsável por coletar os sinais gerados pelos canais de leituras dos calorímetros e prover medidas de energia das partículas incidentes. $\mathrm{O}$ segundo, HLT (do inglês, High Level Trigger), é implementado em software distribuído e usa as informações fornecidas pelo L1 para identificar as partículas de interesse [12], [15]. A Fig. 2 ilustra o HLT para as cadeias responsáveis por identificação de fótons.

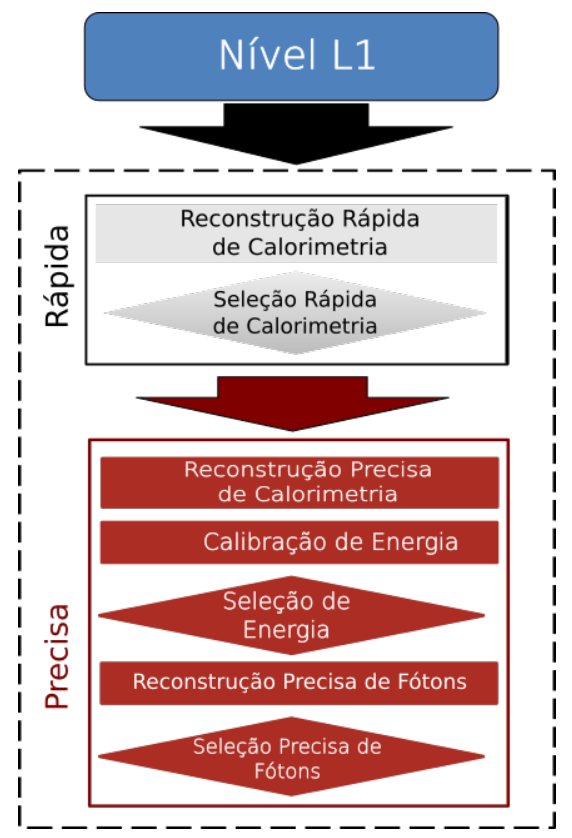

Figura 2: Sistema de seleção de eventos em cadeias de fótons no experimento ATLAS (adaptada de [12]

O L1 entrega informações de energia e posição da partícula incidente para a etapa Rápida do HLT. Esta, por sua vez, faz uma avaliação rápida das variáveis de calorimetria e decide se aquela partícula é um candidato a fóton. Caso a etapa rápida decida por rejeitar um determinado candidato, toda a informação relacionada a esse evento é descartada, reduzindo o custo computacional atribuído para a etapa Precisa. Essa última, por sua vez, opera com etapas de calibração, reconstrução e seleção de partículas que serão posteriormente analisadas de maneira offline, caso o candidato a fóton seja aprovado também por essa etapa [12].

As variáveis utilizadas pelo atual sistema de seleção de fótons são baseadas em medidas de energia como: razão de energia hadrônica, espalhamento lateral da energia depositada pela partícula, dentre outros. Conforme a taxa de colisões próton-próton, ou o número médio de interações por cruzamento de feixes, aumenta, maior a probabilidade da ocorrência de empilhamento de sinais lidos eletrônicamente no calorímetro. Quando isso ocorre, os métodos de estimação de energia operam de forma sub-ótima, comprometendo as medidas de calorimetria utilizadas pela etapa Rápida e Precisa. Como consequência direta desse fenômeno, o desempenho 
dos algoritmos presentes nestas etapas é também degradado [12]. Dessa forma, busca-se constantemente que os algoritmos do sistema de trigger do experimento ATLAS tornem-se resilientes ao empilhamento de sinais.

O sistema de seleção de fótons opera com três critérios: o mais rigoroso, tight, o menos rigoroso, loose e um nível intermediário denominado de medium. $\mathrm{O}$ critério tight, quando aprova um candidato a fóton, indica que aquela partícula possui uma alta probabilidade de ser um fóton. Já quando um candidato é rejeitado pelo critério loose, o sistema de trigger sinaliza que aquela partícula não é um fóton. Entretanto, há o cenário em que uma partícula é rejeitada pelo critério tight e aprovada pelo loose. Essa situação é usada quando deseja-se gerar uma alta estatística na busca de eventos raros.

\section{B. Algoritmo NeuralRinger}

$\mathrm{O}$ algoritmo NeuralRinger foi originalmente proposto em [11], como uma alternativa aos métodos usuais de identificação de elétrons em sistemas de calorimetria. O algoritmo, como mostrado na Fig. 3, monta anéis concêntricos utilizando como centro a célula que possui maior energia (em vermelho).

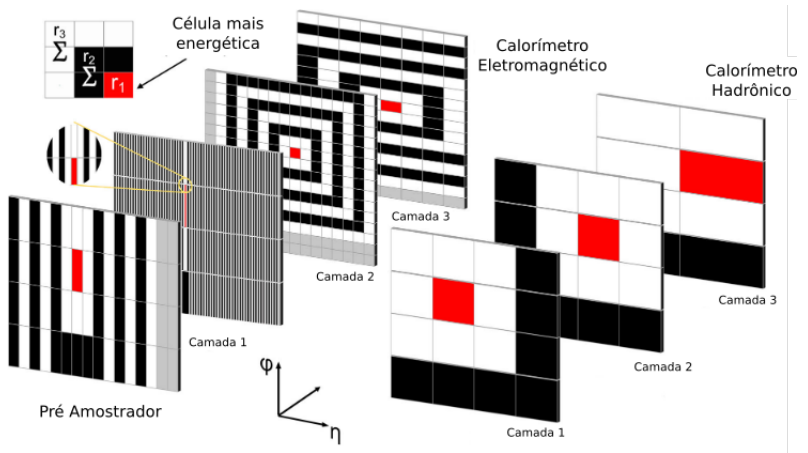

Figura 3: Construção dos anéis em cada camada do sistema de calorimetria (extraído de [12])

O algoritmo então monta os anéis no PS (do inglês, PreSampler), nas três camadas do calorímetro eletromagnético (E1-E3) e para as nas três camadas do calorímetro hadrônico (H1-H3). A quantidade de anéis montados em cada camada é detalhada na Tabela 【

Tabela I: Quantidade de anéis por camada do sistema de calorimetria do experimento ATLAS

\begin{tabular}{|c|c|c|c|c|c|c|c|}
\hline Camadas & PS & EM1 & EM2 & EM3 & H1 & H2 & H3 \\
\hline Anéis & 8 & 64 & 8 & 8 & 4 & 4 & 4 \\
\hline
\end{tabular}

Em seguida, as células pertencentes a cada anel são somadas, gerando um total de 100 medidas de energia que capturam, indiretamente, informação de lateralidade e profundidade do espalhamento de energia depositada pela partícula durante a interação da mesma com a matéria. Essas medidas são então concatenadas em um vetor que alimenta um classificador binário.
A partir de 2017, o algoritmo NeuralRinger tornou-se a técnica padrão de identificação na etapa Rápida nas cadeias de elétrons no experimento ATLAS, operando com um classificador neural MLP [7]. A estrutura da rede neural utilizada para este cenário consiste em apenas uma camada escondida e um neurônio na camada de saída, a fim de realizar a discriminação elétron/jato.

O experimento ATLAS hoje opera com um conjunto de redes neurais, para diversas faixas de energia, $E_{T}$, e posição, $\eta$, do sistema de calorimetria [16], [17]. O sucesso do NeuralRinger aplicado ao problema de classificação de elétrons é uma combinação do alto poder discriminante da estrutura em anéis aliado a eficiência do uso de redes neurais MLP em problemas de classificação binária. Devido ao fato de elétrons e fótons possuírem comportamento similares do ponto de vista de calorimetria [13], esse trabalho propõe a adaptação do algoritmo NeuralRinger para a identificação de fótons no experimento ATLAS.

\section{MÉTODO}

A primeira etapa realizada para o desenvolvimento deste trabalho consistiu em coletar as assinaturas de fótons e jatos hadrônicos. Esses dados foram coletados a partir de dados simulados pela colaboração ATLAS, considerando a verdade desta associada a cada candidato, seguindo-se para a separação destes no plano $E_{T} \times|\eta|$. Com os dados agrupados neste plano, procedeu-se ao treinamento dos modelos neurais, seguindo para a seleção do melhor modelo através do processo de validação cruzada. Uma vez obtido o melhor modelo para operar, procede-se com o procedimento de correção do limiar de decisão por valores de empilhamento. A fim de avaliar a performance do método proposto, a eficiência na identificação de fótons é contabilizada emulando o sistema de seleção de eventos online do ATLAS.

A adaptação do NeuralRinger para identificação rápida de fótons segue uma metodologia similar à desenvolvida para o caso de elétrons. O conjunto de dados utilizado para treinamento e teste dos modelos neurais consiste em fótons isolados para a classe de sinal e qualquer outra partícula para a classe de jatos. Esses dados são advindos de simulação de Monte Carlo, onde são simuladas colisões com decaimentos que resultam em fótons e jatos hadrônicos, colhendo a informação de energia para cerca de 200 mil canais de leitura. Os dados foram separados, no plano $\eta \times E_{T}$, em 25 regiões definidas na Tabela II.

Tabela II: Regiões do plano $\eta \times E_{T}$ utilizadas no desenvolvimento do NeuralRinger para fótons

\begin{tabular}{|c|c|c|c|c|c|}
\hline$E_{T}[\mathrm{GeV}]$ & {$[15,20]$} & {$[20,30]$} & {$[30,40]$} & {$[40,50]$} & {$[50, \infty)$} \\
\hline$|\eta|$ & {$[0 ; 0,8]$} & {$[0,8 ; 1,37]$} & {$[1,37 ; 1,54]$} & {$[1,54 ; 2,37]$} & {$[2,37 ; 2,5]$} \\
\hline
\end{tabular}

A estrutura do modelo adotada foi a MLP, de maneira similar ao realizado para o caso de elétrons. Os modelos possuem apenas uma camada escondida e um neurônio na camada de saída, utilizando neste último a função de ativação $\tanh (\cdot)$. 
Dessa forma, para os candidatos a sinal foram atribuídos a saída +1 e aos jatos foi atribuído a saída -1 . Visando obter um modelo de menor complexidade possível, devido às restrições de tempo imposta pelo regime de colisões do LHC, a quantidade de neurônios da camada escondida foi variada de 2 a 5 .

Para avaliar o desempenho do modelo, utiliza-se o índice soma-produto [18], [19]:

$$
S P=\sqrt{\sqrt{P_{D}\left(1-P_{F}\right)} \frac{P_{D}+\left(1-P_{F}\right)}{2}}
$$

em que $P_{D}$ é a probabilidade de detecção medida sobre a classe de sinal e $P_{F}$ é a probabilidade de falso alarme medida sobre a classe de jato. O índice $S P$ é uma interessante medida para avaliar o desempenho de um modelo através de medidas diretas associadas à qualidade da classificação. O procedimento de treinamento adotado utiliza como critério de parada a maximização do índice $S P$, o $S P_{\max }$. Os parâmetros de treinamento estão sumarizados na Tabela [II

Tabela III: Parâmetros de treinamento do NeuralRinger

\begin{tabular}{c|c}
\hline Inicializações & 5 \\
\hline Função custo (loss) & Mean Square Error (MSE) \\
\hline Parada & $S P_{\max }$ \\
\hline Otimizador & Adam \\
\hline Paciência & 25 \\
\hline Validação Cruzada & $k$-Fold estratificado $(k=10)$ \\
\hline
\end{tabular}

Devido ao fato de o empilhamento de sinais degradar a eficiência na identificação de partículas, após o treinamento é realizada a correção por empilhamento. Esse procedimento consiste em, após obter o melhor modelo para cada região do plano $\eta \times E_{T}$, remover a função de ativação da camada de saída e tornar o limiar de decisão da rede neural como função do empilhamento da amostra. Atualmente, essa correção é feita de maneira linear, seguindo a relação:

$$
\sigma(\langle\mu\rangle)=a\langle\mu\rangle+b
$$

em que $\langle\mu\rangle$ é o empilhamento médio da amostra e $\sigma$ é o limiar de decisão da rede neural. Os coeficientes $a$ e $b$ são ajustados no procedimento de correção do empilhamento. O diagrama na Fig. 4 ilustra o processo de identificação de fótons divididos em todas as 25 regiões do plano $\eta \times E_{T}$.

Uma vez que este trabalho ambienta-se no experimento ATLAS, o impacto do NeuralRinger foi avaliado nas cadeias de fótons através de uma emulação destas, a fim de verificar se a probailidade de falso alarme é reduzida entre a etapa Rápida e a etapa Precisa. Foi verificado também se a eficiência de todo o sistema de filtragem é mantida, bem como a redução de falsos candidatos a fótons aprovados pelo HLT.

\section{RESUltados}

Como resultado da seleção dos sinais através da verdade da simulação e consequente separação dos dados no plano $\eta \times E_{T}$, obteve-se a quantidade de candidatos a fóton e a jato em cada uma das regiões, mostrado na Fig. 5 .

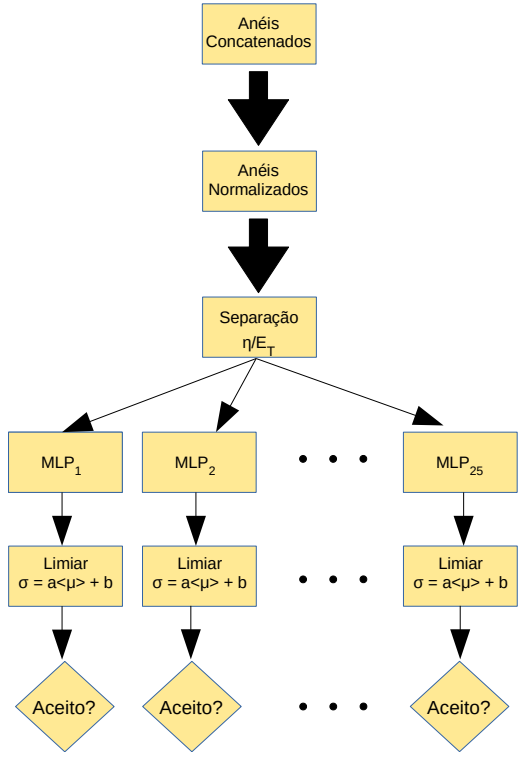

Figura 4: Diagrama ilustrativo da operação do conjunto de

\begin{tabular}{|c|c|c|c|c|c|}
\hline \multirow{2}{*}{${ }_{4: 2.37 \times 2.50}$} & \multicolumn{5}{|c|}{ Estatística disponível de fóton/jato } \\
\hline & $f: 15328$ & $f: 20874$ & $f: 24438$ & $f: 27064$ & $f: 144706$ \\
\hline \multirow{3}{*}{$3: 1.54>2.37$} & $\mathrm{j}: 303375$ & j : 317697 & j : 151205 & $\mathrm{j}: 72070$ & j: $: 72088$ \\
\hline & $f: 109202$ & $f: 139161$ & $f: 184213$ & $f: 184407$ & $f: 969941$ \\
\hline & j : 2257068 & $\mathrm{j}: 2367100$ & $\mathrm{j}: 1109731$ & j : 518850 & j : 505414 \\
\hline \multirow{2}{*}{$2: 1.37>>1.54$} & $f: 19440$ & $f: 25974$ & $f: 40707$ & $f: 45605$ & $f: 174407$ \\
\hline & $\mathrm{j}: 410499$ & $\mathrm{j}: 453386$ & j : 219728 & $\mathrm{j}: 102531$ & j : 102198 \\
\hline \multirow{2}{*}{$1: 0.80 \cdot>1.37$} & $f: 76160$ & $f: 104684$ & $f: 127603$ & $f: 134628$ & $f: 694101$ \\
\hline & $j: 1725472$ & $j: 1767962$ & j : 778466 & $\mathrm{j}: 351064$ & j : 347076 \\
\hline \multirow{2}{*}{$0: 0.00 \cdot \times 0.80$} & $f: 117166$ & $f: 145389$ & $f: 191923$ & $f: 189824$ & $f: 987678$ \\
\hline & j : 2776105 & j : 2827188 & j : 1255151 & j : 563416 & j : 551373 \\
\hline
\end{tabular}
redes neurais com correção de empilhamento.

Figura 5: Quantidade de fótons e jatos para as diferentes regiões de operação do NeuralRinger.

Já a Fig. 6 mostra a distribuição estatística dos fótons e jatos com relação a energia e a posição $\eta$ do sistema de coordenadas. Nota-se, na Fig. 6a em que a região $|\eta| \approx 1,4$ existe uma queda na contagem de partículas. Isso se deve ao fato de que, nessa região, a instrumentação do calorímetro eletromagnético possui uma resolução menor do que as demais regiões. Isso ocorre devido a passagem de cabos e a limitações na montagem do calorímetro. Já a Fig. 6b mostra que a quantidade de fótons supera a de jatos. Isso é devido ao fato de que, em energias elevadas, aumenta-se a relação sinal-ruído. As ondulações presentes no histograma de fótons são devidas ao uso de quatro conjunto de dados distintos para extrair os sinais associados a essa partícula. Cada um deles possui uma especificação mínima de energia $(17,35,50$ e $70 \mathrm{GeV})$ e é onde se concentra a maior parte dos sinais, gerando assim um pico na contagem em torno desses valores.

A Fig. 7 mostra o perfil dos sinais coletados para fótons e jatos. Observa-se que nos anéis finais (camadas hadrônicas) a energia dos jatos é, em média, superior a de fótons. Já nos 


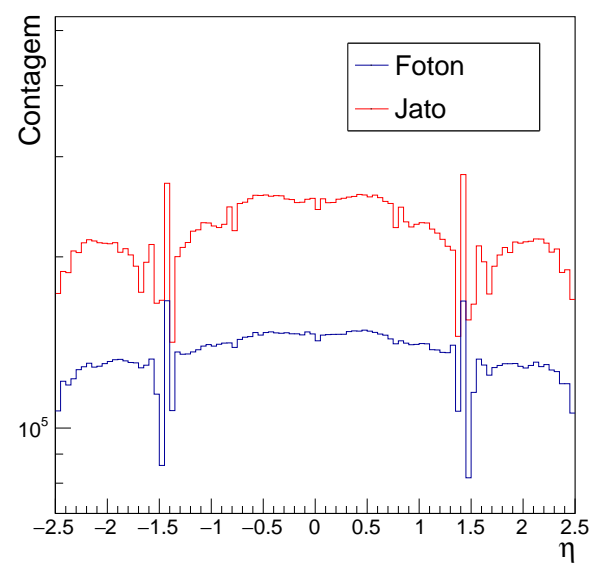

(a)

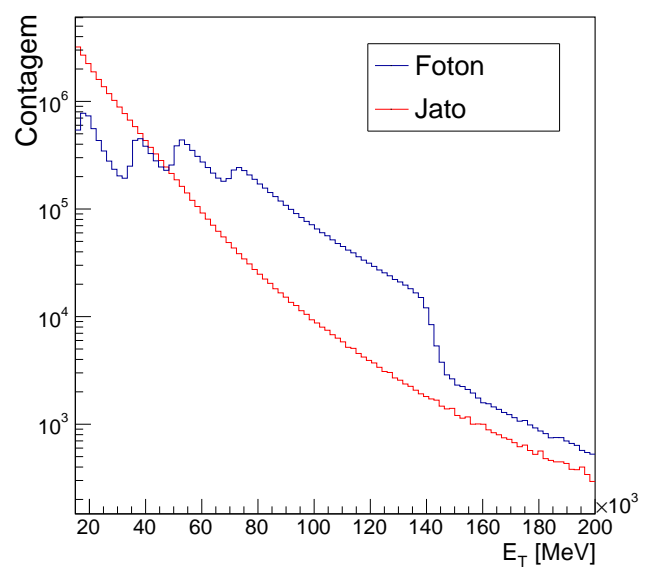

(b)

Figura 6: Histogramas dos fótons e jatos com relação a (a): posição. (b): energia.

primeiros anéis, nota-se que a energia dos fótons é superior a dos jatos. Essa característica gerada pelo procedimento de montagem dos anéis confere alto poder discriminante entre as classes exploradas nesse trabalho.

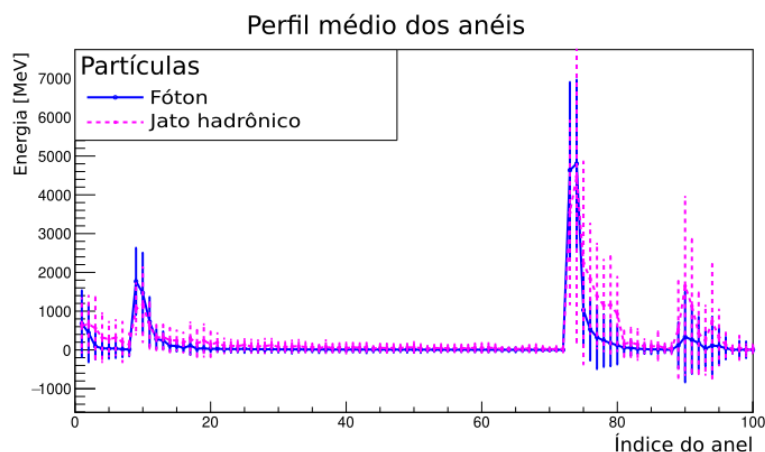

Figura 7: Perfil médio do vetor de anéis para fótons e jatos hadrônicos.
A Fig. 8 mostra a avaliação do resultado do treinamento de um modelo de 2 neurônios na camada oculta para uma única região de operação do NeuralRinger. Na Fig. 8a é mostrada a evolução do MSE durante a fase de treinamento e a fase de teste. Nota-se que ambas as curvas, vermelha e azul, possuem o mesmo comportamento, indicando que não há nenhum tipo de sobre-ajuste durante a etapa de treinamento.

A linha vertical preta indica a época em que se obteve o máximo índice $S P$, este mostrado na Fig. 8b Como mencionado na Tabela III, o treinamento tenta ainda por 25 épocas encontrar um novo $S P_{\max }$. Nesse caso, após a época 26 não foi possível encontrar e, portanto, esta foi a melhor época selecionada durante o treinamento. É válido ressaltar que comportamento similar foi observado nas demais regiões de operação e para os modelos com mais neurônios na camada oculta.

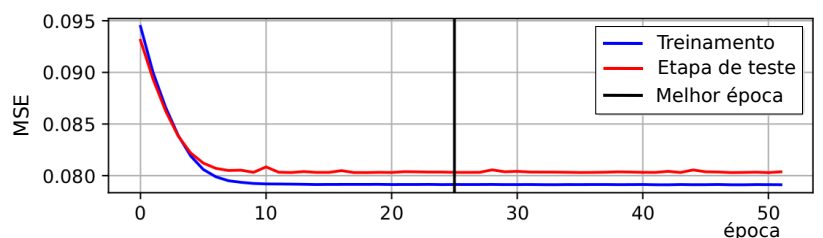

(a)

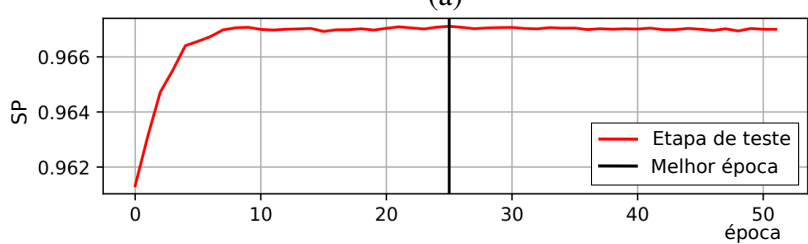

(b)

Figura 8: (a): Evolução do MSE ao longo das épocas durante a fase de treinamento e fase de teste, avaliado sobre um modelo de 2 neurônios na camada oculta, no quarto subconjunto da validação cruzada. Esse modelo foi treinado usando os dados da região $0,8<|\eta|<1,37$ e $20<E_{T}<30 \mathrm{GeV}$. (b): Evolução do índice SP para a mesma região de operação e mesmo modelo.

O resultado da avaliação de modelos com diferentes quantidades de neurônios na camada oculta, para a região explorada, é mostrado na Tabela IV] comparando com o atual método de seleção baseado cortes lineares (Referência). Aqui, a probabilidade de detecção dos modelos é fixada no mesmo ponto da referência, a fim de avaliar o impacto no falso alarme.

Tabela IV: Comparação de diferentes modelos com o atual método de seleção de fótons $\left(20<E_{T}<30 \mathrm{GeV}\right.$ e $0,8<$ $|\eta|<1,37)$ para o critério medium.

\begin{tabular}{lcc}
\hline & $P_{D}[\%]$ & $P_{F}[\%]$ \\
\hline Referência & 94,24 & 25,88 \\
MLP 2 Neurônios & $94,24 \pm 0,00$ & $13,46 \pm 0,06$ \\
MLP 3 Neurônios & $94,24 \pm 0,00$ & $12,94 \pm 0,03$ \\
MLP 4 Neurônios & $94,24 \pm 0,00$ & $13,04 \pm 0,04$ \\
MLP 5 Neurônios & $94,24 \pm 0,00$ & $12,84 \pm 0,06$
\end{tabular}


Em geral, os modelos reduzem em aproximadamente $45 \%$ a probabilidade de falso alarme com relação ao atual método de seleção. Nota-se também que, quando comparado com o método de referência, o NeuralRinger reduz drasticamente a taxa de falso alarme, mantendo a mesma probabilidade de deteç̧ão, ou seja, sem prejuízo na seleção de fótons verdadeiros. Observa-se também que, ao aumentar a quantidade de neurônios da camada oculta, não há uma redução significativa na taxa de falso alarme. Por essa razão, o modelo com o menor número de neurônios da camada oculta é escolhido, uma vez que apresenta a menor complexidade computacional, fator crucial para ambientes com alta taxa de eventos. Uma das razões para tanto se dá pelo alto poder discriminativo associado a montagem dos anéis para fótons e jatos.

A correção por empilhamento é mostrada na Fig. 9. Nos gráficos mostrados nesta, é destacado o ajuste da reta por valores de empilhamento, bem como a concentração de candidatos a fótons e jatos, mostrados nas Fig. 9a e 9b Os pontos (em azul escuro) são posicionados para manter o mesmo $P_{D}$ para todas as faixas de empilhamento. Nesse caso, ol $P_{D}$ foi ajustado para $94.4 \%$, mesmo valor obtido pelo atual método de seleção rápida nas cadeias de fótons no experimento ATLAS para essa região de operação. Nota-se, na Fig. 9a que a maior quantidade de sinais está a direita da reta ajustada pelos pontos, indicando que a maioria dos sinais foram corretamente classificados pelo modelo. De maneira similar, observa-se na Fig. 9b que a maioria das partículas que compõe os jatos estão a esquerda da mesma reta, indicando que estes também foram corretamente classificados pelo NeuralRinger.

Afim de avaliar o impacto da substituição da atual técnica de seleção rápida de fótons pelo NeuralRinger, algumas cadeias de seleção comparando ambos os métodos foram emuladas, considerando todo o HLT. As emulações foram feitas sobre os dados de fótons e de jatos, para avaliar eficiência e redução de falso alarme. $\mathrm{O}$ resultado dessa emulação, considerando a classe de sinal, é mostrado na Fig. 10. As emulações foram feitas considerando diferentes tipos de seleção. $\mathrm{O}$ corte em energia é indicado pelo valor seguinte à letra $g$ (g20, por exemplo, seleciona fótons a partir de $20 \mathrm{GeV}$ ). O critério de pureza da amostra (tight, medium ou loose) é indicado ao lado do corte em energia. Uma cadeia denominada, por exemplo, $H L T \_g 20 \_t i g h t$ seleciona fótons a partir de $20 \mathrm{GeV}$ que foram aprovados pelo critério tight do sistema HLT;

Observa-se que a eficiência do HLT foi mantida para os três pontos de operação do trigger para toda a faixa de energia. A Fig. 11 mostra também a eficiência medida com relação ao empilhamento da amostra não foi alterada. Já a Fig. 12 mostra que a eficiência é mantida também para quase todas as faixas de $\eta$, com exceção da região próxima do final do detector $|\eta| \approx 2.5$. Nessa região, como pode-se observar, nenhum fóton é aprovado pelo critério tight, indicando que essa região é problemática para identificação desse tipo de partícula. No ATLAS, a manutenção dos níveis de eficiência de todo o sistema de seleção de eventos é de suma importância.

A eficiência do método contabilizada em jatos é mostrada nas Fig. 13, 14, 15 e 16, A Fig. 13 mostra a aceitação de falsos

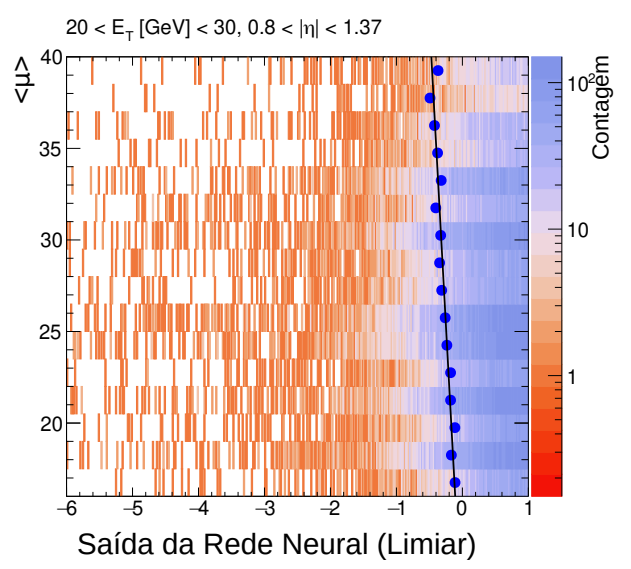

(a)

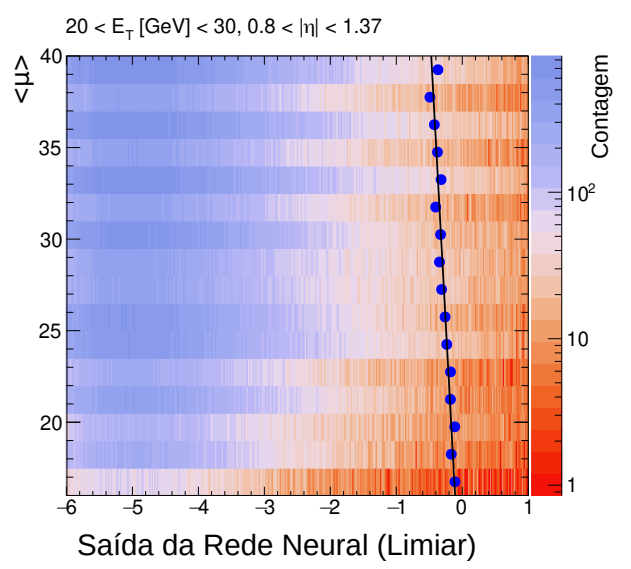

(b)

Figura 9: Correção por empilhamento considerando as distribuições de(a): fótons (b): jatos

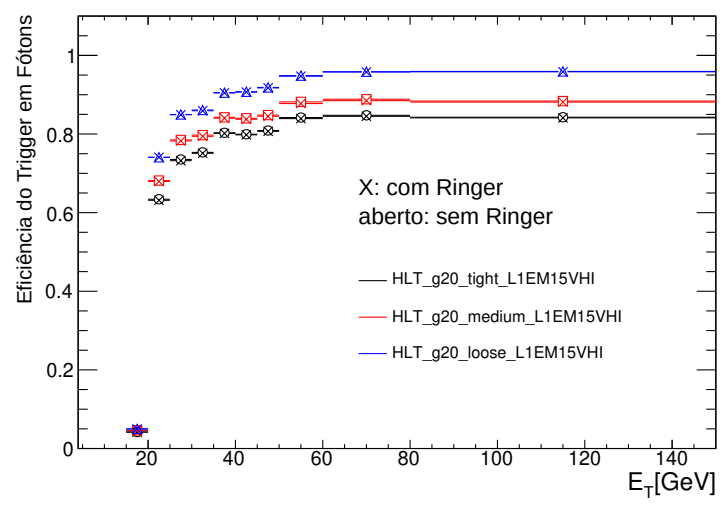

Figura 10: Análise de eficiência do HLT medida sobre a classe de sinal com relação a energia, considerando os critérios Tight, Medium e Loose 


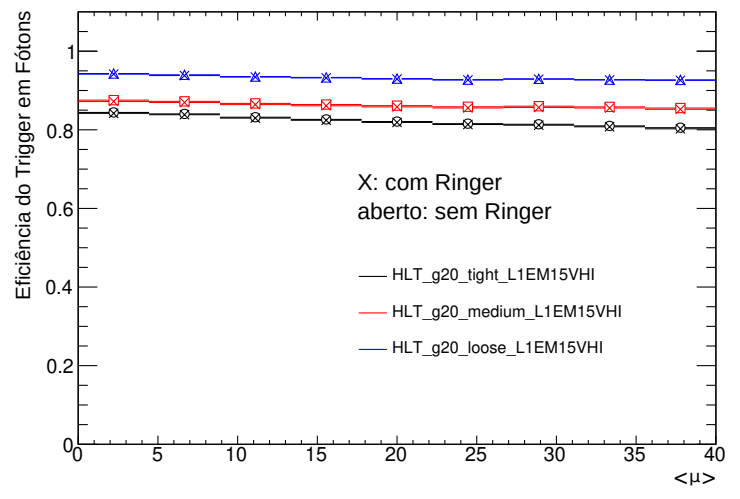

Figura 11: Análise de eficiência do HLT medida sobre a classe de sinal com relação ao empilhamento, considerando os critérios Tight, Medium e Loose

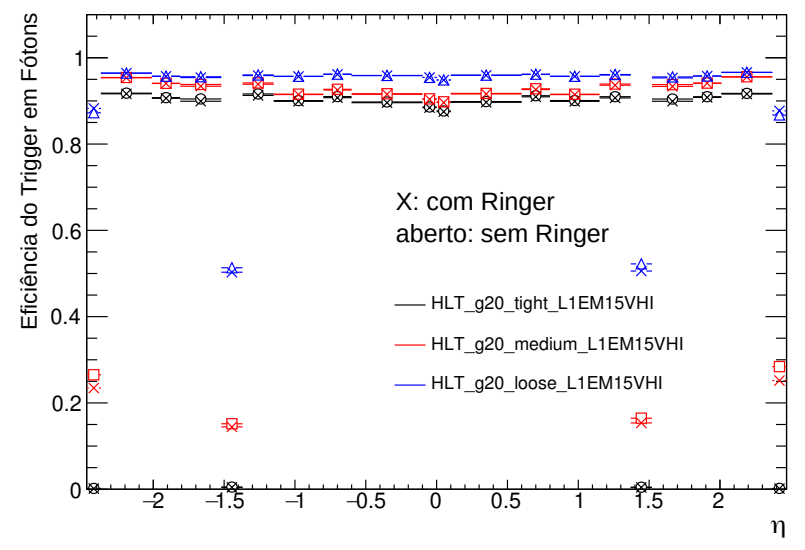

Figura 12: Análise de eficiência do HLT medida sobre a classe de sinal com relação a coordenada $\eta$, considerando os critérios Tight, Medium e Loose

fótons por faixas de energia. Observa-se que, para todos os pontos de operação, o Neural Ringer rejeita uma quantidade maior de jatos quando comparado a estratégia de seleção baseada em cortes lineares. Na Fig. 14 nota-se o mesmo fato por faixas de empilhamento de sinais. Devido ao processo de correção por empilhamento, nota-se que as curvas associadas ao NeuralRinger apresentam comportamento quase constante. Já na Fig. 15 observa-se também que o NeuralRinger rejeita uma maior quantidade de jatos por valores de $\eta$. Nota-se também uma queda drástica em $\eta \approx 1.5 \mathrm{em}$ virtudes dos fatores já mencionados. Por sua vez, a Fig. 16 mostra a taxa de aceitação de falsos fótons contabilizada ao final de todo o sistema de filtragem online de eventos. Nela, nota-se uma ligeira redução do falso alarme quando comparado com o atual método de seleção de fótons no experimento ATLAS.

Nota-se ainda os critérios tight, medium e loose apresentam a mesma eficiência nas Fig. 13, 14 e 15. Isso se dá pelo fato de, atualmente, os cortes nas variáveis de calorimetria são os

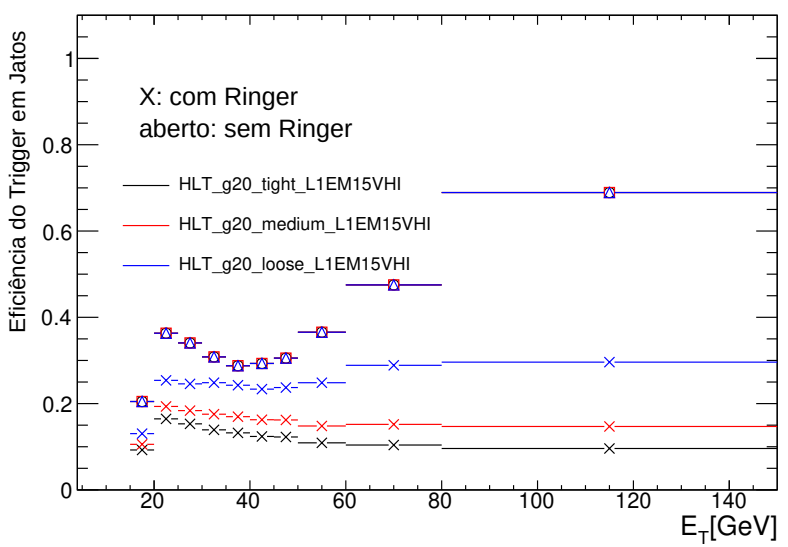

Figura 13: Análise de eficiência da etapa Rápida medida sobre os jatos com relação energia, considerando os critérios Tight, Medium e Loose

mesmos para os três critérios. A consequência da redução da taxa de aceitação de falsos fótons, quando o NeuralRinger é utilizado na etapa Rápida, é a redução no custo computacional atribuído à etapa Precisa, que é crítico dado o regime de operação de alta taxa de colisões do LHC.

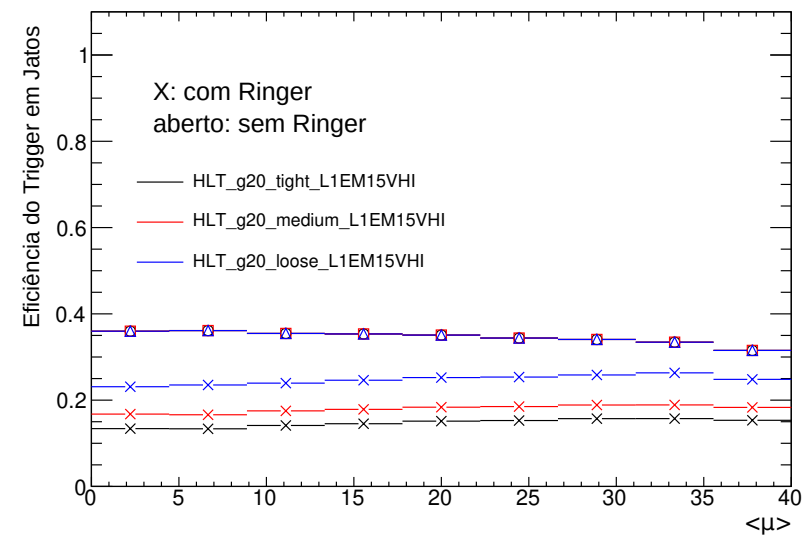

Figura 14: Análise de eficiência da etapa Rápida medida sobre jatos com relação ao empilhamento, considerando os critérios Tight, Medium e Loose

Já na Fig. 16 os três critérios apresentam desempenhos distintos, uma vez que essa medida é feita em todo o sistema de trigger, incluindo a etapa Precisa. Nessa análise, nota-se a redução. Deve-se destacar que a redução é significativa, haja vista a alta taxa de eventos. Dessa forma, a frequência de com que os eventos são salvos para análise offline é reduzida, poupando os requisitos de armazenamento e facilitando a busca pelos raros eventos de interesse.

As análises mostradas aqui evidenciam que a técnica NeuralRinger aplicada para fótons mantém a eficiência no sistema de seleção online de eventos e reduz a aceitação de 


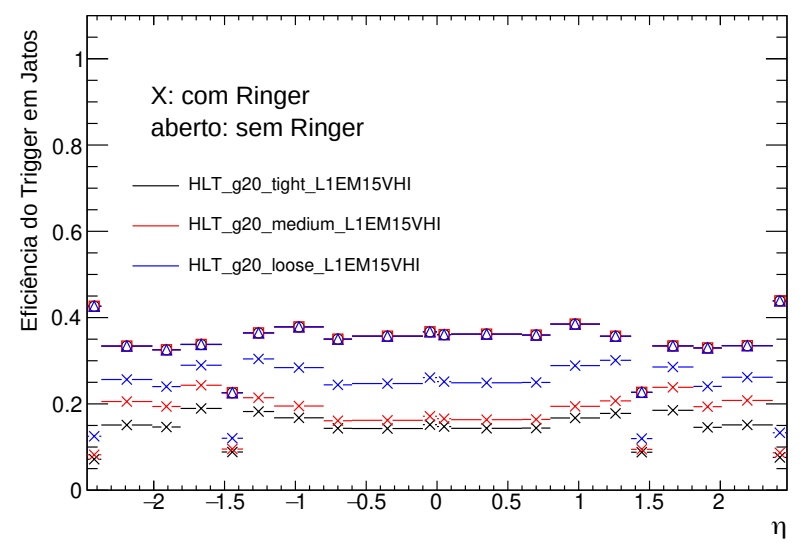

Figura 15: Análise de eficiência da etapa Rápida medida sobre os jatos com relação a coordenada $\eta$, considerando os critérios Tight, Medium e Loose

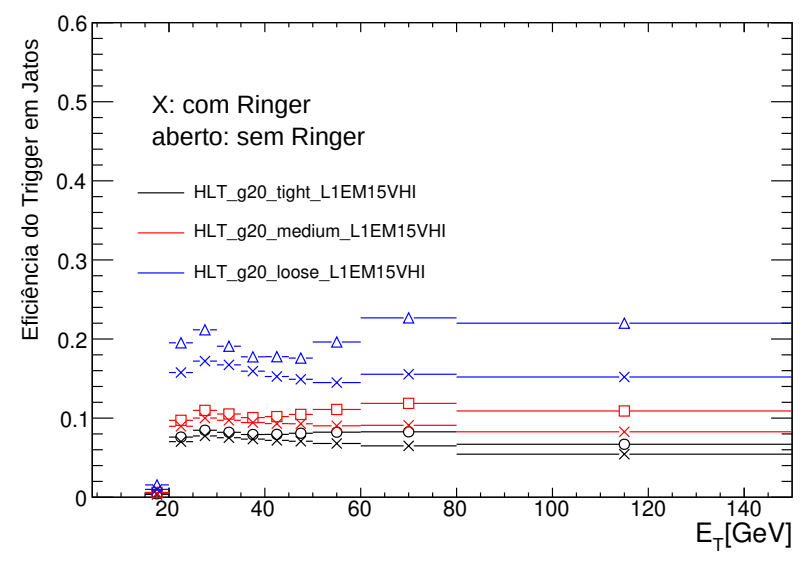

Figura 16: Análise de eficiência do HLT medida sobre os jatos com relação a energia, considerando os critérios Tight, Medium e Loose

jatos quando comparada com a técnica atual do experimento ATLAS. Dessa forma, a técnica poupa custo computacional das etapas posteriores à etapa rápida e diminui a escrita em disco ao final do processamento dos eventos.

\section{Conclusões}

O experimento ATLAS é o maior do LHC, contribuindo significativamente para diversas descobertas científicas. Neste experimento, diversas técnicas são aplicadas para a rápida identificação de partículas. Dentre elas, o algoritmo NeuralRinger, que faz uso de redes neurais artificiais, mostra-se uma interessante abordagem nesse contexto. A adaptação desta técnica para a detecção rápida de fótons exibe potencial na aplicação do experimento, uma vez que reduz a ocorrência de falsos positivos entre as etapas do HLT e na geração dos arquivos para posterior análise offline. Dessa forma, para a próxima etapa de atualizações do LHC, espera-se que o
NeuralRinger de fótons opere como técnica padrão nas cadeias de processamento deste tipo de partícula.

\section{AGRADECIMENTOS}

Os autores agradecem a FAPESB, CNPq, FAPEMIG, FAPERJ e RENAFAE pelo apoio financeiro e à colaboração ATLAS pelas contribuições técnicas ao trabalho. Agradecem também ao IFBA campus Vitória da Conquista. O presente trabalho foi realizado com apoio da Coordenação de Aperfeiçoamento de Pessoal de Nível Superior - Brasil (CAPES) - Código de Financiamento 001.

\section{REFERÊNCIAS}

[1] D. J. Griffiths, Introduction to elementary particles; 2nd rev. version. Physics textbook, New York, NY: Wiley, 2008.

[2] L. Evans and P. Bryant, "LHC machine," Journal of Instrumentation, vol. 3, pp. S08001-S08001, aug 2008.

[3] ATLAS Collaboration, "The ATLAS experiment at the CERN Large Hadron Collider," Journal of Instrumentation, S08003 (2008), 012008.

[4] I. De Lotto and D. Dotti, "Pile-up effects in time measurements," Nuclear Instruments and Methods, vol. 39, no. 2, pp. 281 - 292, 1966.

[5] ATLAS Collaboration, "Observation of a new particle in the search for the standard model Higgs boson with the ATLAS detector at the LHC,' Physics Letters B, vol. 716, no. 1, pp. 1 - 29, 2012.

[6] G. Aad, B. Abbott, et al., "Evidence for higgs boson decays to a lowmass dilepton system and a photon in pp collisions at $\sqrt{s}=13 \mathrm{TeV}$ with the atlas detector," Physics Letters B, vol. 819, p. 136412, 2021.

[7] S. S. Haykin, Neural networks and learning machines. Upper Saddle River, NJ: Pearson Education, third ed., 2009.

[8] A. Radovic, M. Williams, D. Rousseau, M. Kagan, D. Bonacorsi, A. Himmel, A. Aurisano, K. Terao, and T. Wongjirad, "Machine learning at the energy and intensity frontiers of particle physics," Nature, vol. 560, 082018.

[9] C. Shimmin, P. Sadowski, P. Baldi, E. Weik, D. Whiteson, E. Goul, and A. Søgaard, "Decorrelated jet substructure tagging using adversarial neural networks," Physical Review D, vol. 96, 032017.

[10] P. Baldi, P. Sadowski, and D. Whiteson, "Searching for exotic particles in high-energy physics with deep learning," Nature communications, vol. 5 , p. 4308, 072014.

[11] J. Seixas, L. Caloba, M. Souza, A. Braga, and A. Rodrigues, "Neural second-level trigger system based on calorimetry," Computer Physics Communications, vol. 95, no. 2, pp. 143-157, 1996.

[12] G. Aad, Abbott, et al., "Performance of electron and photon triggers in atlas during lhc run 2," Eur. Phys. J. C, vol. 80, p. 47.56 p, Sep 2019.

[13] R. Wigmans, Calorimetry: Energy Measurement in Particle Physics. International series of monographs on physics, Clarendon Press, 2017.

[14] ATLAS Collaboration, "Technical Design Report for the Phase-II Upgrade of the ATLAS TDAQ System,” Tech. Rep. CERN-LHCC-2017020. ATLAS-TDR-029, CERN, Geneva, Sep 2017.

[15] A. Ruiz-Martinez and A. Collaboration, "The Run-2 ATLAS Trigger System," tech. rep., CERN, Geneva, Feb 2016.

[16] J. V. da Fonseca Pinto and the ATLAS Colaboration, "An ensemble of neural networks for online filtering implemented in the ATLAS trigger system," Journal of Physics: Conference Series, vol. 1162, p. 012039, jan 2019.

[17] W. Spolidoro Freund, "An Ensemble of Neural Networks for Online Electron Filtering at the ATLAS Experiment," J. Phys. Conf. Ser., vol. 1525, no. 1, p. 012076, 2020.

[18] R. C. Torres, D. E. F. de Lima, E. F. de Simas Filho, and J. M. de Seixas, "Neural online filtering based on preprocessed calorimeter data," in 2009 IEEE Nuclear Science Symposium Conference Record (NSS/MIC), pp. 530-536, 2009.

[19] E. F. Simas Filho, J. M. de Seixas, and L. P. Calôba, "Modified postnonlinear ica model for online neural discrimination," Neurocomputing, vol. 73, no. 16, pp. 2820-2828, 2010. 10th Brazilian Symposium on Neural Networks (SBRN2008).

[20] D. Kingma and J. Ba, "Adam: A method for stochastic optimization," International Conference on Learning Representations, 122014. 